\title{
NANOCOMPÓSITOS DE POLIURETANA TERMOPLÁSTICA E NANOTUBOS DE CARBONO DE PAREDES MÚLTIPLAS PARA DISSIPAÇÃO ELETROSTÁTICA
}

\author{
Rodrigo L. Lavall, Juliana A. de Sales, Raquel S. Borges, Hallen D. R. Calado, José C. Machado, Dario Windmöller e \\ Glaura G. Silva* \\ Departamento de Química, Instituto de Ciências Exatas, Universidade Federal de Minas Gerais. Av. Antônio Carlos, 6627,
} 31270-901 Belo Horizonte - MG, Brasil

Rodrigo G. Lacerda e Luiz O. Ladeira

Departamento de Física, Instituto de Ciências Exatas, Universidade Federal de Minas Gerais. Av. Antônio Carlos, 6627, 31270-901 Belo Horizonte - MG, Brasil

Recebido em 9/3/09; aceito em 14/7/09; publicado na web em 27/11/09

\begin{abstract}
THERMOPLASTIC POLYURETHANE AND MULTI-WALLED CARBON NANOTUBES NANOCOMPOSITES FOR ELECTROSTATIC DISSIPATION. Polyurethane/multi-walled carbon nanotube (MWCNT) nanocomposites have been prepared with nanotube concentrations between $0.01 \mathrm{wt} \%$ and $1 \mathrm{wt} \%$. MWCNT as-synthesized samples with $\sim 74 \mathrm{~nm}$ diameter and $\sim 7 \mathrm{~m}$ length were introduced by solution processing in the polyurethane matrix. Scanning electron microscopy (SEM) images demonstrated good dispersion and adhesion of the CNTs to the polymeric matrix. The $\mathrm{C}=\mathrm{O}$ stretching band showed evidence of perturbation of the hydrogen interaction between urethanic moieties in the nanocomposites as compared to pure TPU. Differential scanning calorimetry and positron anihilation lifetime spectroscopy measurements allowed the detection of glass transition displacement with carbon nanotube addition. Furthermore, the electrical conductivity of the nanocomposites was significantly increased with the addition of CNT.
\end{abstract}

Keywords: thermoplastic polyurethane; carbon nanotube; nanocomposites.

\section{INTRODUÇÃO}

Os nanotubos de carbono (NTC) apresentam combinação única de propriedades mecânicas, elétricas e térmicas que os tornam excelentes candidatos para substituir ou complementar as cargas convencionais utilizadas no preparo de nanocompósitos poliméricos. Por exemplo, os nanotubos de carbono de parede única, denominados SWCNT da sigla em inglês, podem ser metálicos ou semicondutores, possuem módulo de Young entre 640 GPa-1TPa, resistência a tração de 150-180 GPa e condutividade térmica teórica de $6000 \mathrm{~W} / \mathrm{mK}$. Os MWCNT's (nanotubos de carbono de paredes múltiplas) possuem características elétricas entre metal e semicondutor, apresentam módulo de Young de 0,27-0,95 TPa, resistência à tração de 11-63 GPa e condutividade térmica entre 200-3000 W/mK (MWCNT isolados). ${ }^{1-3}$ Adicionalmente, os NTC possuem alta flexibilidade, baixa densidade mássica e alta razão comprimento/diâmetro (tipicamente entre 300-1000).

A alta razão comprimento/diâmetro e o elevado número de interações de van der Waals entre os nanotubos de carbono levam à formação de agregados. O grande desafio no campo de nanocompósitos polímero/nanotubo de carbono é a dispersão dos nanotubos em tubos individuais (ou agregados de poucos tubos) nas matrizes poliméricas, ${ }^{3,4}$ principalmente quando altas concentrações de nanotubos são empregadas.

As poliuretanas (PU) são uma classe de materiais poliméricos com importância industrial significativa, por possuírem grande variedade de aplicações como espumas, adesivos, borrachas, entre outras. Uma PU típica é um polímero produzido por reação de poliadição de um isocianato (di ou polifuncional) com um poliol (polióis-poliéteres são os mais utilizados) e outros reagentes, principalmente agentes de cura ou extensores de cadeia. Na PU termoplástica (TPU), sem reticulação, os extensores de cadeia reagem com o di-isocianato para formar os segmentos rígidos. Os polióis dão origem aos segmentos

*e-mail: glaura@qui.ufmg.br flexíveis, que são geralmente incompatíveis com os segmentos rígidos, ocasionando separação de fases (segregação). Os segmentos rígidos são os responsáveis pelas propriedades termomecânicas das PU's, enquanto que os segmentos flexíveis controlam as propriedades de flexibilidade a baixas temperaturas e resistência a solventes.

No presente trabalho foram preparados nanocompósitos poliméricos com nanotubos de carbono de paredes múltiplas (MWCNT) e poliuretana termoplástica (TPU) no intuito de obter aumento significativo de condutividade elétrica em relação à matriz com o emprego de pequenas quantidades da carga. Dissipação eletrostática em materiais poliméricos pode ser alcançada através da adição de cargas condutoras e, no presente, a adição de baixas concentrações de nanocargas constitui uma estratégia especialmente interessante. ${ }^{5}$ Esta propriedade é importante em diversas aplicações de plásticos e borrachas, como nas indústrias automobilística e aeronáutica. ${ }^{6}$ Valores de condutividade superiores a $10^{-6} \mathrm{~S} / \mathrm{cm}$ devem ser obtidos para garantir dissipação de carga. ${ }^{5}$ Os materiais preparados foram caracterizados quanto à sua estrutura por técnicas espectroscópicas, buscando estabelecer correlações estrutura versus propriedades de interesse tecnológico.

$\mathrm{O}$ estado da arte com respeito a estes nanomateriais indica duas principais abordagens para o preparo de nanocompósito polímero/ nanobubo, a saber, baseadas nas ligações covalentes e nas interações não covalentes polímero-nanotubos. ${ }^{3,7,8}$ Em abordagens baseadas em interação não-covalente, como nos compósitos preparados nesse trabalho, vários estudos demonstraram que o polímero preferencialmente se enrola ao redor do nanotubo (wrapping). No entanto, Smalley e colaboradores ${ }^{8}$ destacam que o polímero não precisa estar necessariamente enrolado ao redor dos tubos em uma conformação regular ou simétrica, mas pode simplesmente recobrir os mesmos impedindo a reagregação.

É bem conhecido que várias propriedades físicas são diretamente influenciadas pela presença de defeitos e "vazios" (vacâncias) verificados em abundância em sistemas de dimensão reduzida. ${ }^{9}$ A 
espectroscopia de aniquilação de pósitrons (EAP) é uma ferramenta versátil na detecção e monitoramento da evolução de defeitos do tipo vacâncias em sistemas sólidos, ${ }^{10}$ com presença cada vez mais marcante no estudo de materiais poliméricos. ${ }^{11,12}$ Compósitos poliméricos com nanotubos de carbono foram alvo de estudos recentes utilizando as técnicas de EAP. ${ }^{9,13}$ Dentre essas técnicas, a espectroscopia de vida média do pósitron (EVMP) permite monitorar as dimensões e concentração de volumes livres (vacâncias) através da obtenção de parâmetros de tempos de vida médios $(\tau)$ e intensidades relativas (I) de formação de espécies positrônicas, respectivamente.

Nosso grupo tem reportado trabalhos utilizando a espectroscopia de vida média do pósitron (EVMP) para obter informações estruturais de sistemas poliméricos complexos. ${ }^{11,12}$ Esta técnica permite acessar como a distribuição de volume livre evolui com a temperatura, por exemplo. ${ }^{14}$ Neste trabalho a medida de EVMP em função da temperatura fornecerá uma sonda sub-nanométrica para avaliar uma importante transição de fase do material polimérico e dos nanocompósitos.

\section{PARTE EXPERIMENTAL}

\section{Produção dos nanocompósitos TPU/MWCNT}

Os nanocompósitos foram produzidos utilizando-se como matriz polimérica uma poliuretana termoplástica aromática à base de poliéter comercial (TPU 990R - Bayer Material Science ${ }^{15}$ ) e nanotubos de carbono de paredes múltiplas (MWCNT) sintetizados pelo grupo do Laboratório de Nanomateriais do Departamento de Física da UFMG por CVD, utilizando ferroceno como catalisador em metodologia típica usada na área. ${ }^{16}$ Os nanotubos foram utilizados como recebidos e apresentam diâmetro médio de 73,5 nm e comprimento médio de $7,4 \mu \mathrm{m}$, determinados por MEV (o comprimento foi obtido de 16 nanotubos isolados e não curvos em regiões de baixa concentração da amostra, enquanto que o diâmetro foi acessado de 55 nanotubos). A imagem MEV apresentada na Figura 1S, material suplementar, é típica da amostra utilizada e mostra a presença de nanopartículas, além de nanotubos. A amostra foi analisada também por termogravimetria (TA Instruments SDT $2960 \mathrm{em}$ ar a $5^{\circ} \mathrm{C} / \mathrm{min}$ ) e análise elementar por EDS (microssonda eletrônica JEOL JSM 840A). O material que é denominado MWCNT contém aproximadamente $15 \%$ de partículas metálicas de ferro encapsuladas por carbono amorfo e grafítico e, talvez, também no interior dos nanotubos. ${ }^{17}$ No presente trabalho optou-se por não realizar procedimentos químicos para retirada deste resíduo metálico por duas razões principais: i) as manipulações químicas de retirada do metal são agressivas e trabalhosas, danificam os nanotubos e apresentam baixo rendimento. ${ }^{18}$ É desejado que as propriedades eletrônicas dos nanotubos sejam conservadas sem a alteração na estrutura $\mathrm{sp}^{2}$ e a conjugação dos átomos de carbono do tubo $^{19}$ causada por esses procedimentos; ii) no caso específico da aplicação visada, partes plásticas com propriedade de dissipação eletrostática, a presença de partículas metálicas encapsuladas por carbono pode não ter impacto negativo potencial, embora este aspecto necessite ser estudado em maiores detalhes. A presença de carbono amorfo e grafítico também não compromete o uso do nanomaterial dentro dos objetivos aqui colocados, sendo que estas partículas podem conduzir carga, apesar da razão de aspecto diferente em relação aos nanotubos. Amostras de nanotubos de carbono do mesmo lote que está sendo utilizado foram estudadas em detalhe pelo nosso grupo e concluiu-se que a concentração de nanotubos de carbono era de aproximadamente $54 \%$ em massa. ${ }^{20}$ Informações complementares sobre este nanomaterial podem ser encontradas na referência 20 e permitem que outros trabalhos sobre a influência de nanotubos de carbono em compósitos poliméricos possam ser comparados com o que será apresentado.
Análises de RMN ${ }^{1} \mathrm{H}$ e ${ }^{13} \mathrm{C}$ (espectrômetro Bucker DPX 200 MHz Advance, com amostra solubilizada em N,N-dimetilformamida deuterada) da TPU comercial (não apresentadas) indicam que se trata de TPU baseada no isocianato 4,4-difenilmetano di-isocianato (MDI) e principalmente no poliéter poli(tetrametilenoglicol) (PTMG) com aproximadamente $14 \%$ de segmentos rígidos e $86 \%$ de segmentos flexíveis. A Figura 2S, material suplementar, mostra a estrutura básica deste tipo de poliuretana.

Neste trabalho, a técnica empregada para o preparo dos nanocompósitos TPU/MWCNT foi a mistura em solução, considerada mais efetiva quando são utilizadas cargas de dimensões muito pequenas ${ }^{3}$ e envolveu três passos básicos: dispersão do nanotubo em THF, mistura com a solução do polímero (à temperatura ambiente) e recuperação do compósito por evaporação do solvente (casting) com obtenção do filme.

No primeiro passo, a dispersão dos MWCNT em THF foi realizada com o emprego de ultrassom a $40 \%$ da potência por $20 \mathrm{~min}$ (ultrassom de ponteira Ultrasonic Processor modelo CPX 130 da Cole Parmer). O ultrassom é necessário para dispersão moderada dos nanotubos em solvente, porque promove a separação dos tubos. ${ }^{3}$ Antes do tratamento com ultrassom, observa-se sedimentação dos nanotubos no fundo do frasco (Figura 1a). As suspensões obtidas após o tratamento são estáveis por cerca de 4 h não ocorrendo sedimentação de material, o que começa a acontecer após esse tempo (Figura 1b). (a)

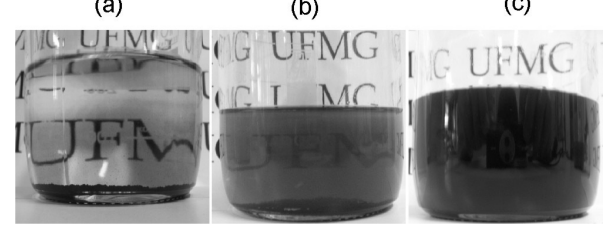

Figura 1. Suspensões do MWCNT em (a) THF antes do ultrassom, (b) THF após ultrassom e 6 h de repouso e (c) em TPU/THF após ultrassom e cerca de 12 em repouso

A TPU foi dissolvida em $50 \mathrm{~mL}$ de THF e, após solubilização, foi misturada à suspensão com nanotubo, antes de qualquer sedimentação, no segundo passo. A suspensão TPU/MWCNT foi então submetida ao ultrassom por $20 \mathrm{~min}$ (40\% da potência). Essa suspensão é estável por mais de $12 \mathrm{~h}$ (Figura 1c), quando começa ser observada sedimentação do material. Foram produzidas dispersões nas seguintes concentrações de nanotubo (em massa): 0,01; 0,1 e 1\% (denominados CP001, CP01 e CP1, respectivamente).

\section{Preparo das membranas}

Para o preparo dos filmes por evaporação do solvente, as suspensões foram transferidas para placas de Petri e estas recobertas com um Becker e deixadas na capela (desligada) por $12 \mathrm{~h}$ para evaporação lenta do THF. Depois de preparados, os filmes foram secos em estufa a vácuo a $30{ }^{\circ} \mathrm{C}$ por 5 dias para eliminação de solvente residual. Na Figura $3 \mathrm{~S}$, material suplementar, são mostradas as imagens desses filmes.

\section{Caracterização}

Os espectros de infravermelho dos filmes das amostras foram adquiridos por transmissão ou refletância (HATR), com resolução de $4 \mathrm{~cm}^{-1}$ e 64 varreduras no espectrofotômetro Perkin-Elmer FTIR Spectrum GX (UFMG). A microscopia eletrônica de varredura (MEV) foi realizada com $\mathrm{V}=15 \mathrm{kV}, \mathrm{i}=6 \times 10^{-11} \mathrm{~A}$, em secção transversal dos filmes recobertos com fina camada de $\mathrm{Au}(10 \mathrm{~s})$ no microscópio Jeol JXA 8900RL (UFMG/CDTN-CNEN). A calorimetria exploratória 
diferencial (DSC) foi realizada em cadinho de alumínio fechado a $10^{\circ} \mathrm{C} / \mathrm{min}$ em atmosfera de $\mathrm{He}(50 \mathrm{~mL} / \mathrm{min})$ em três varreduras consecutivas: 25 até $200 ; 200$ até $-120 ;-120$ até $200^{\circ} \mathrm{C}$ no equipamento TA Instruments 2920 DSC. A espectroscopia de impedância (EI) foi utilizada a $0 \mathrm{~V}$ com amplitude de $50 \mathrm{mV}$ na faixa de frequência compreendida entre $1 \times 10^{6}$ e $0,5 \mathrm{~Hz}$ no potenciostato/galvanostato Ecochemie Autolab PGSTAT30. Para as medidas eletroquímicas os materiais foram secos cuidadosamente em estufa/dessecador sob vácuo. A espessura das camadas foi verificada utilizando-se medidor de espessura digital com precisão de $1 \mu \mathrm{m}$ sob mesa de medição de alta exatidão da Mitutoyo. Os filmes apresentaram espessuras de: 87 (TPU), 123 (CPTPU001), 164 (CPTPU01) e 68 m (CPTPU1) e área constante determinada pela área do coletor de corrente $29,24 \mathrm{~mm}^{2}$.

As medidas de espectroscopia de vida média do pósitron (EVMP) foram efetuadas em atmosfera de ar, na faixa de temperatura entre 21 e $90^{\circ} \mathrm{C}$, em um sistema de coincidência rápido-rápido (ORTEC), com resolução temporal de $260 \mathrm{ps}$. Como fonte de pósitrons foi utilizado ${ }^{22} \mathrm{NaCl}$ (Amersham) colocado entre duas folhas de Kapton de $7 \mu \mathrm{m}$ de espessura. A atividade da fonte era de aproximadamente $22 \mu \mathrm{Ci}$. Filmes de cada material foram empilhados até ser obtida espessura de 0,65 mm e, no meio dos blocos (de 0,65 mm cada), foi colocada a fonte de pósitrons numa configuração do tipo "sanduíche". Os espectros de tempo de vida foram analisados com três componentes, utilizando-se o programa Positronfit Extend, ${ }^{21}$ que forneceu os parâmetros tempo de vida $\left(\tau_{\mathrm{i}}\right)$ e intensidade relativa $\left(\mathrm{I}_{\mathrm{i}}\right)$, em que $\mathrm{i}=1$, 2,3 , representam as espécies p-Ps, $\mathrm{e}^{+}$e o-Ps, respectivamente. Para os tempos de vida obtidos, a componente mais curta $\left(\tau_{1}=0,125\right)$ foi associada ao p-Ps e mantida fixa na análise dos espectros. A análise com esse parâmetro livre forneceu resultados semelhantes, dentro do erro experimental da medida. Os tempos de vida, determinados na análise do espectro experimental, são valores médios da distribuição de tempos de vida das espécies positrônicas. De acordo com o modelo do volume livre, ${ }^{22}$ é possível correlacionar o tempo de vida médio do o-Ps com a dimensão dos volumes livres utilizando a expressão semi-empírica

$\tau_{3}=\frac{1}{\lambda_{3}}=\frac{1}{2}\left[1-\frac{R}{R_{0}}+\frac{1}{2} \pi \operatorname{sen}\left(2 \pi \frac{R}{R_{0}}\right)\right]^{-1}$

em que $\mathrm{R}_{\mathrm{o}}=\mathrm{R}+\Delta \mathrm{R}$. e $\Delta \mathrm{R}$ é um parâmetro empírico determinado como sendo igual a $1,66 \AA^{23}$

$\mathrm{O}$ volume livre, $\mathrm{V}_{l}$, é determinado com o auxílio da Equação 2, considerando que esses espaços "vazios" tenham forma esférica

$V_{l}=\frac{\left(4 \pi R^{3}\right)}{3}$

\section{RESULTADOS E DISCUSSÃO}

A maior estabilidade da suspensão obtida com os nanotubos adicionados de TPU (Figura 1c) indica que ocorre um mecanismo semelhante ao observado por Shvartzman-Cohen e colaboradores, ${ }^{24}$ que empregaram copolímeros bloco (de poliéteres) para dispersão de nanotubos de carbono em soluções aquosas ou em solventes orgânicos. Esses pesquisadores verificaram que a utilização de ultrassom, em condições brandas, favorecia a separação dos feixes de nanotubos com a formação de nanotubos individuais, os quais por seletiva adsorção dos copolímeros blocos seriam mantidos isolados, devido à repulsão estérica entre as unidades individuais de CNT/copolímero bloco. ${ }^{24}$ Os "blocos" da TPU (segmentos rígidos e flexíveis) provavelmente interagem com os nanotubos e os mantêm separados por tempo suficiente para a deposição dos filmes, como representado esquematicamente na Figura 2.

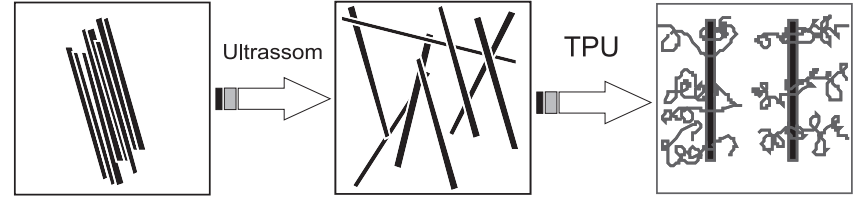

Figura 2. Representação simplificada do processo de dispersão dos nanotubos de carbono com o auxílio de sonicação e na presença de poliuretana

\section{Microscopia eletrônica de varredura (MEV)}

Imagens por MEV da superfície de fratura foram adquiridas para todos os filmes no intuito de verificar a microestrutura nos compósitos TPU/MWCNT (Figura 3). (a)

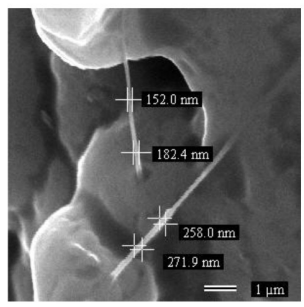

(c)

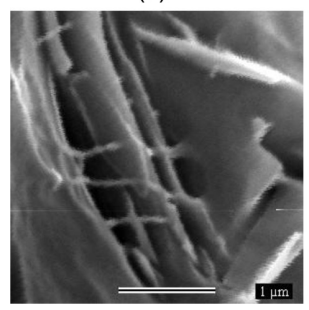

(b)

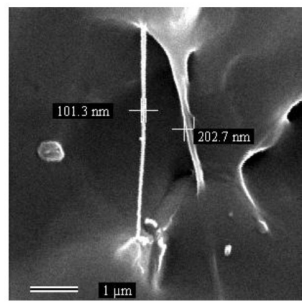

(d)

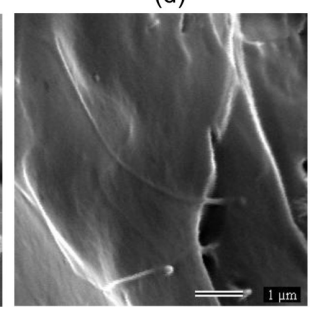

Figura 3. Imagens selecionadas de superficie de fratura de diferentes compósitos: (a) CP001; (b) CP01; (c) e (d) CP1

As imagens mostram a morfologia típica verificada em todos os compósitos nos quais os nanotubos estão bem dispersos pela matriz. Pode-se observar nanotubos isolados recobertos pela matriz polimérica em toda a faixa de concentração estudada. A variação no diâmetro, numa faixa bem maior que a verificada para o nanotubo puro, sugere o recobrimento por polímero. Os tubos apresentam boa adesão à matriz, conectando as trincas (Figura $3 \mathrm{c}$ ).

Todas as imagens indicam que o procedimento empregado gerou uma microestrutura propícia à obtenção de boas propriedades mecânicas e elétricas nos compósitos.

\section{Calorimetria exploratória diferencial (DSC)}

As curvas DSC dos nanocompósitos são similares àquela da TPU pura mostrada na Figura 4S, material suplementar. Os valores das propriedades térmicas, retirados da segunda corrida de aquecimento da DSC são apresentados na Tabela 1.

Pelos dados da Tabela 1 pode-se verificar a presença de duas Tg's para a TPU em $-35^{\circ} \mathrm{C}\left(\mathrm{Tg}_{1}\right)$ e $61^{\circ} \mathrm{C}\left(\mathrm{Tg}_{2}\right)$, relacionadas aos segmentos

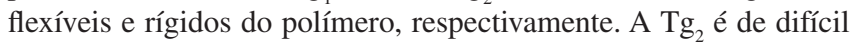
visualização quando é utilizada DSC convencional, mas foi confirmada por dados de espectroscopia de vida média de pósitron (EVMP) (discutidos posteriormente). Na Figura 4S, material suplementar, é mostrado igualmente o procedimento adotado para a determinação da segunda transição vítrea, que é precedida em geral por uma inflexão na linha de base. Esta inflexão na linha de base pode estar associada 
Tabela 1. Propriedades térmicas, para TPU e nanocompósitos, retiradas da segunda corrida de aquecimento

\begin{tabular}{lccc}
\hline Amostra & $\mathrm{T}_{\mathrm{g}}($ midpoint $)\left({ }^{\circ} \mathrm{C}\right)$ & $\mathrm{T}_{\text {fus }}($ no pico $)\left({ }^{\circ} \mathrm{C}\right)$ & $\Delta_{\text {fus }} \mathrm{H}(\mathrm{J} / \mathrm{g})$ \\
\hline $\mathrm{TPU}$ & -35 e 61 & $115^{*} / 168$ & 6 \\
$\mathrm{CP001}$ & -37 e 59 & $118 * / 169$ & 5 \\
$\mathrm{CP01}$ & -37 e 56 & $101 * / 168$ & 5 \\
$\mathrm{CP} 1$ & -36 e 56 & 167 & 4 \\
\hline
\end{tabular}

* ombro

a um alargamento substancial da faixa de temperatura da transição em questão ou a sobreposição de fenômenos oriundos dos segmentos flexíveis, como relatado por Koerner e colaboradores. ${ }^{25}$

Uma transição endotérmica em $168{ }^{\circ} \mathrm{C}$ (com ombro em $113{ }^{\circ} \mathrm{C}$ ) com calor da ordem de 6,2 J/g, foi observada. De acordo com van Heumen e Stevens, ${ }^{26}$ dados de DSC indicam que além da Tg a baixas temperaturas, relacionada aos segmentos flexíveis, poliuretanas com concentrações de segmentos rígidos consideráveis (superior a 15\% em massa) podem apresentar até três transições endotérmicas. A primeira $\left(\mathrm{t}_{1}\right)$, em temperaturas na ordem de $70-100^{\circ} \mathrm{C}$ está relacionada a ordenamento de curto alcance (short-range) dos segmentos rígidos; a segunda $\left(\mathrm{t}_{2}\right)$, na faixa de temperatura de $120-170{ }^{\circ} \mathrm{C}$ é devida ao ordenamento de longo alcance (long-range) e a terceira $\left(\mathrm{t}_{3}\right)$, para poliuretanas com altas concentrações de segmentos rígidos, acontecem em temperaturas superiores a $200^{\circ} \mathrm{C}$ e está relacionada à microcristalinidade apresentada pelos segmentos rígidos. ${ }^{26} \mathrm{De}$ acordo com os dados, observaram-se para a TPU 990R os eventos relacionados ao ordenamento de curto e longo alcances dos segmentos rígidos (que estão se sobrepondo), com baixa absorção de calor (Figura 4S, material suplementar).

Os compósitos apresentam duas Tg's relacionadas aos segmentos flexíveis (em valores da ordem de $-35^{\circ} \mathrm{C}$ ) e rígidos da TPU. Pode-se verificar que o MWCNT induz ligeira diminuição na Tg relacionada aos segmentos rígidos. Esse resultado pode ser explicado por interações entre os nanotubos e os segmentos rígidos da poliuretana, melhor evidenciado por mudanças na banda de estiramento $\mathrm{C}=\mathrm{O}$ nos dados de infravermelho (discutidos a seguir). Duas transições endotérmicas descritas para a TPU, relacionadas com ordenamento de curto alcance $\left(t_{1}\right)$ e ordenamento de longo alcance $\left(t_{2}\right)$ dos segmentos rígidos, foram observadas em todos os compósitos.

\section{Espectroscopia vibracional na região do infravermelho}

Foram adquiridos espectros na região do infravermelho da TPU e dos compósitos e as principais bandas foram atribuídas (Tabela 2).

Os modos de deformação axial NH do isocianato e deformação axial C-O-C do poliéter são típicos da estrutura da TPU. Da mesma forma, os modos de deformação angular fora do plano, na região em torno de $800 \mathrm{~cm}^{-1}$, estão associados aos grupos aromáticos do MDI. Para os compósitos, não foram observadas bandas adicionas ou deslocamentos consideráveis em relação à TPU pura.

Segundo tem sido reportado, as bandas em 3503 e $3328 \mathrm{~cm}^{-1}$, próprias de deformação axial do grupo N-H e as bandas em 1731 e $1700 \mathrm{~cm}^{-1}$, características da carbonila, podem ser empregadas para o estudo do grau de interação entre os segmentos rígidos e flexíveis de poliuretanas. ${ }^{27-30} \mathrm{~A}$ natureza das ligações de hidrogênio presentes na estrutura de diferentes TPU's tem sido investigada segundo o procedimento descrito por Digar e Wen ${ }^{27}$ e Wen e colaboradores, ${ }^{28,29}$ com base nas bandas de deformação axial $\mathrm{NH}$, e pelo que foi proposto por Lee et al., ${ }^{30}$ com o emprego das bandas de deformação axial $\mathrm{C}=\mathrm{O}$. Os procedimentos estão baseados na deconvolução das bandas características utilizando função Gaussiana. ${ }^{30} \mathrm{~A}$ deconvolução da banda de deformação axial $\mathrm{C}=\mathrm{O}$ é empregada, considerando-se o estiramento $\mathrm{C}=\mathrm{O}$ livre em torno de $1731 \mathrm{~cm}^{-1}$ (banda 1) e o estiramento $\mathrm{C}=\mathrm{O}$ participando de ligações de hidrogênio em cerca de $1700 \mathrm{~cm}^{-1}$ (banda 2). Os grupos carbonila uretânicos livres $\left(1731 \mathrm{~cm}^{-1}\right)$ e interagindo por ligações de hidrogênio $\left(1700 \mathrm{~cm}^{-1}\right)$ são associados, respectivamente, às ligações de hidrogênio entre grupos uretânicos e o poliéter e às ligações de hidrogênio entre grupos uretânicos. ${ }^{30}$

Esse procedimento foi empregado para analisar a TPU Texin 990R e para verificar possíveis modificações devido à presença dos MWCNTs, através de ajustes empregando função Gaussiana (Figura 4). Para tanto, as áreas das bandas foram normalizadas em relação à área total da banda característica de cada deformação. Dessa forma, a área em porcentagem tabelada (Tabela 3 ) relaciona quantitativamente, com base na contribuição total do modo de estiramento característico, cada tipo de interação presente na estrutura da TPU. As áreas de cada pico (Tabela 3) mostram um maior número de ligações de hidrogênio entre grupos uretânicos na TPU pura (74\%), indicando grande separação de fases no material de partida. Lee e colaboradores, ${ }^{30}$ aplicando o procedimento descrito, verificaram para uma das TPU's estudadas valores da ordem destes obtidos para a Texin 990R (Tabela 3) com área da banda 1 igual a 65,7\% e área da banda 2 igual a 24,9\%.

Tabela 2. Tentativa de atribuição de modos vibracionais e respectivos números de onda para a TPU ${ }^{28,29,40}$ e nanocompósitos

Número de onda $\left(\mathrm{cm}^{-1}\right) /$ amostra

\begin{tabular}{|c|c|c|c|c|c|}
\hline TPU & CP001 & $\mathrm{CP} 01$ & $\mathrm{CP} 1$ & Grupo funcional & Modo vibracional \\
\hline 3503 & 3501 & 3503 & 3502 & $\mathrm{NH}$, livre * & $v$ \\
\hline 3327 & 3326 & 3327 & 3326 & NH, lig. de hidrogênio & $v$ \\
\hline 2935,2921 & 2938 & 2936 & 2935 & $\mathrm{CH}_{2}$, alifático & $v_{a}$ \\
\hline 2852 & 2856 & 2850 & 2851 & $\mathrm{CH}_{2}$, alifático & $v_{\mathrm{s}}$ \\
\hline 1731 & 1729 & 1729 & 1729 & $\mathrm{C}=\mathrm{O}$, livre & $v_{\mathrm{s}}$ \\
\hline 1700 & 1699 & 1700 & 1699 & $\begin{aligned} \mathrm{C}=\mathrm{O}, \text { lig. de hi- } & \\
& \text { drogênio }\end{aligned}$ & $v_{\mathrm{s}}$ \\
\hline 1596,1528 & 1596 & 1596 & 1596 & $\mathrm{C}=\mathrm{C}$, aromático & $v$ \\
\hline 1221 & 1220 & 1220 & 1220 & $\mathrm{C}-\mathrm{O}-\mathrm{C}$ & $v_{\mathrm{a}}$ \\
\hline 1079 & 1075 & 1077 & 1075 & $\mathrm{C}-\mathrm{O}-\mathrm{C}$ & $v_{\mathrm{s}}$ \\
\hline $\begin{array}{l}816,771, \\
749\end{array}$ & 816,770 & 817,771 & 816,770 & $\mathrm{C}-\mathrm{H}$, aromático & $\bar{\omega}$ \\
\hline
\end{tabular}

v (deformação axial ou estiramento); $v_{\mathrm{a}}$ (deformação axial assimétrica); $v_{\mathrm{s}}$ (deformação axial simétrica); $\varpi$ (deformação angular fora do plano); * pequeno ombro. 
Mais interessante é o fato experimental que, com a adição dos MWCNTs, bons ajustes só foram possíveis empregando-se três Gaussianas. A terceira Gaussiana aparece em torno de $1672 \mathrm{~cm}^{-1}$. Trata-se de uma linha alargada para todas as composições similar à mostrada na Figura 4 para o CP001 (indicada na figura como B3). Esses dados sugerem que os grupos uretânicos estejam sendo perturbados pela presença dos nanotubos no material. Um aprofundamento da análise da interação entre nanotubos e TPU só poderá ser realizado com medidas complementares, por exemplo, por espalhamento de raios $\mathrm{X}$ em baixo ângulo.
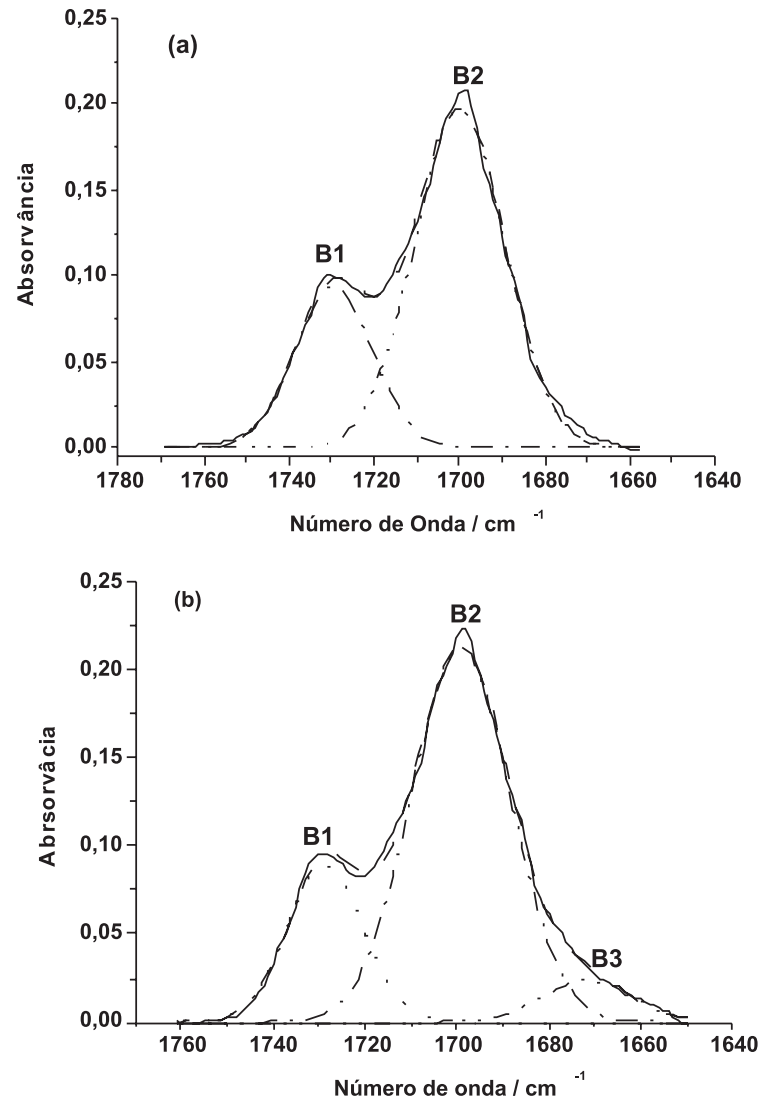

Figura 4. Exemplos do procedimento de ajuste por Gaussianas utilizado para (a) TPU e (b) CPOO1

Tabela 3. Dados obtidos do ajuste da banda de estiramento $\mathrm{C}=\mathrm{O}$ no espectro de IV

\begin{tabular}{lcccccc}
\hline Amostra* & $\begin{array}{c}\mathrm{B} 1 \\
\left(\mathrm{~cm}^{-1}\right)\end{array}$ & $\begin{array}{c}\mathrm{B} 2 \\
\left(\mathrm{~cm}^{-1}\right)\end{array}$ & $\begin{array}{c}\mathrm{B} 3 \\
\left(\mathrm{~cm}^{-1}\right)\end{array}$ & $\begin{array}{c}\mathrm{A} 1 \\
(\%)\end{array}$ & $\begin{array}{c}\mathrm{A} 2 \\
(\%)\end{array}$ & $\begin{array}{c}\mathrm{A} 3 \\
(\%)\end{array}$ \\
\hline TPU & 1731 & 1700 & - & 26 & 74 & - \\
CP001 & 1729 & 1699 & 1672 & 22 & 71 & 7 \\
CP01 & 1729 & 1700 & 1674 & 26 & 70 & 4 \\
$\mathrm{CP} 1$ & 1729 & 1699 & 1671 & 23 & 69 & 8 \\
\hline
\end{tabular}

* B1, B2 e B3 são as posições das bandas (ver Figura 4) após ajuste e A1, A2 e A3 as respectivas áreas normalizadas pela área total.

\section{Espectroscopia de vida média do pósitron (EVMP)}

A EVMP foi empregada no estudo dos compósitos preparados nesse trabalho (Tabela 4). Pode-se observar que, considerando os desvios experimentais, não se observa variação significativa em termos de dimensões dos volumes livres $\left(\tau_{3}(\mathrm{~ns})\right)$ em todas as amostras medidas à temperatura ambiente. Entretanto, quando se analisa o parâmetro ligado à concentração de volumes livres $\left(\mathrm{I}_{3}(\%)\right)$, observa-se que para o compósito $\mathrm{CP} 1$, a concentração de "espaços vazios" é ligeiramente menor, o que pode indicar que uma fração de buracos/defeitos no sistema desaparece (maior compactação) nessa concentração de nanotubo de carbono. Esta observação é confirmada quando se analisa o parâmetro que relaciona os dois parâmetros de aniquilação da espécie orto-positrônica $\left(\mathrm{I}_{3}\right.$ e $\left.\tau_{3}\right)$ : a f (fração de volume livre), dado pela Equação $3,{ }^{31}$ calculando a fração relativa de volumes livres, isto é, fv/C (ver Tabela 4, com cálculos em termos de $\mathrm{nm}^{3}$ ). Olhando a evolução dos valores de $\mathrm{f}_{\mathrm{v}}$ (através de $\mathrm{f}_{\mathrm{v}} / \mathrm{C}$ na Tabela 4), observa-se uma tendência de crescimento até CP01 e, em CP1 ele cai. Chakrabarti e colaboradores ${ }^{9}$ observaram a tendência à diminuição de $\mathrm{I}_{3}$ em compósitos preparados com fibras de poliacrilonitrila com MWCNT em relação à matriz a partir de 1\% (em massa). Eles prepararam compósitos nas concentrações de 1, 3 e 5\% (em massa) de nanotubo para a matriz. ${ }^{9}$

$\mathrm{f}_{\mathrm{v}}=\mathrm{CI}_{3} \mathrm{~V}$

em que C é uma constante característica do sistema polimérico, determinada experimentalmente.

Tabela 4. Parâmetros de aniquilação de pósitrons para TPU e compósitos, obtidos com a técnica EVMP, a $25^{\circ} \mathrm{C}$

\begin{tabular}{|c|c|c|c|c|c|}
\hline Amostras & $\tau_{3}(\mathrm{~ns})$ & $\mathrm{I}_{3}(\%)$ & $\mathrm{R}(\AA)$ & $\mathrm{V}_{l}\left(\AA^{3}\right)$ & $\mathrm{f}_{\mathrm{v}} / \mathrm{C}\left(\mathrm{nm}^{3} \%\right)$ \\
\hline TPU & $2,31 \pm 0,01$ & $29,6 \pm 0,2$ & 3,117 & 126,8 & 3,75 \\
\hline CP $0,01 \%$ & $62,32 \pm 0,30$ & $30,1 \pm 0,3$ & 3,125 & 127,8 & 3,85 \\
\hline CP $0,1 \%$ & $2,32 \pm 0,01$ & $30,0 \pm 0,3$ & 3,125 & 127,9 & 3,84 \\
\hline CP $1 \%$ & $2,33 \pm 0,01$ & $28,9 \pm 0,2$ & 3,131 & 128,5 & 3,71 \\
\hline
\end{tabular}

De especial interesse é o estudo via EVMP da transição de fase associada aos segmentos rígidos na TPU $\left(\mathrm{Tg}\right.$ a $61{ }^{\circ} \mathrm{C}$ segundo a DSC - Tabela 1) e sua variação com a adição dos nanotubos de carbono, através de experimentos em função da temperatura. As medidas foram realizadas na faixa de temperatura entre 25 e $90{ }^{\circ} \mathrm{C}$. Os parâmetros de tempo de vida médio $\left(\tau_{3}\right)$ e intensidade $\left(I_{3}\right)$ estão apresentados na Figura 5.
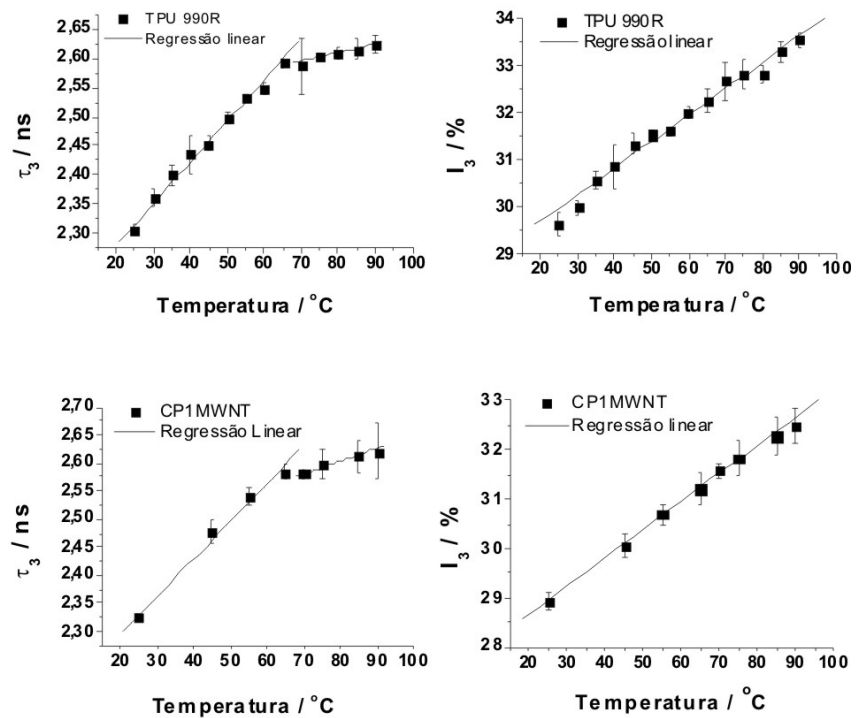

Figura 5. Avaliação da evolução dos parâmetros $\tau_{3}$ e $I_{3}$ com a temperatura: exemplo do polímero puro e compósito com $1 \%$ (em massa) de MWCNT 
Pode-se observar nessa figura que, como esperado, $\tau_{3}$ e $I_{3}$ aumentam com a temperatura. No entanto, o parâmetro $\tau_{3}$ mostra uma inflexão na $\mathrm{Tg}$. Isso é observado tanto no polímero puro quanto nos compósitos preparados, o que permitiu avaliar a Tg. Os valores obtidos são apresentados na Tabela 5.

Tabela 5. Valores de Tg obtidos por EVMP

\begin{tabular}{lc}
\hline Material & $\mathrm{Tg} /{ }^{\circ} \mathrm{C}$ \\
\hline TPU & 64 \\
$\mathrm{CP} 001$ & 55 \\
$\mathrm{CP} 01$ & 58 \\
$\mathrm{CP} 1$ & 58 \\
\hline
\end{tabular}

Esses resultados confirmam os dados obtidos por DSC e a tendência à ligeira diminuição da Tg dos segmentos rígidos da TPU com a adição dos nanotubos. A nanocarga introduzida na TPU produziu uma estrutura dos domínios rígidos que necessita menor quantidade de energia para transitar entre o estado vítreo e elastomérico. Portanto, os nanotubos de carbono estão perturbando os domínios rígidos, como já indicado por espectroscopia no infravermelho.

\section{Espectroscopia de impedância (EI)}

Medidas de EI foram realizadas na faixa de temperatura de 25-95 ${ }^{\circ} \mathrm{C}$. Pode-se verificar, no diagrama de Nyquist para o nanocompósito (exemplo da Figura 6), uma reta deslocada da origem que desenvolve um ângulo próximo a $90^{\circ}$ com o eixo da impedância real (Z'). Esse comportamento foi observado em toda a faixa de temperatura estudada para todos os compósitos. Para a TPU pura obtém-se um semicírculo (Figura 6) associado a comportamento dielétrico com alta resistência ao transporte de carga.

Esses dados foram simulados utilizando um circuito RQ (resistência em paralelo com elemento de fase constante), comumente descrito para compósitos condutor/isolante. ${ }^{32}$ Várias tentativas foram realizadas até que os erros relativos aos parâmetros $(\mathrm{R}, \mathrm{Q}, \mathrm{n})$ ficassem abaixo de $5 \%$ (na maioria dos casos abaixo de 1\%). A condutividade foi calculada com o emprego da Equação 4 e os valores obtidos apresentados no diagrama de Arrhenius da Figura 7.

$C=\frac{l}{A R}$

sendo $l$ a espessura do filme, $A$ área e $R$ a resistência.

Todos os compósitos apresentam valores de condutividade praticamente constantes com a variação de temperatura (o que denota comportamento de condutor eletrônico) e várias ordens de grandeza maiores que a condutividade da matriz (Figura 7) devido à percolação elétrica. O limiar de percolação é caracterizado por um aumento agudo, de várias ordens de grandeza, na condutividade ${ }^{33}$ devido à formação de rede tridimensional infinita das partículas da carga através dos componentes da matriz. Observa-se (Figura 7) que para os nanocompósitos TPU/MWCNT isso acontece em concentrações de nanotubo inferiores ou da ordem de $0,01 \%$. Para compósitos à base de poliuretana, Koerner e colaboradores ${ }^{34}$ reportaram valor de limiar de percolação de $0,85 \%$ (em massa) (compósitos TPU(de poliéster)/MWCNT). Um dos valores de limiar de percolação mais baixos $\left(0,0025 \%\right.$, em massa) foi descrito por Sandler e colaboradores ${ }^{33}$ para compósitos MWCNT/resina epóxi. Musumeci e colaboradores ${ }^{35}$ descreveram que na maioria dos estudos recentes sobre compósitos, nos quais os nanotubos foram dispersos isotropicamente e de forma eficiente na matriz polimérica, existe uma convergência em relação ao limiar de percolação elétrico. Limiares de percolação menores que $1 \%$ (em massa) e frequentemente da ordem de $0,01-0,1 \%$ (em massa) são observados. ${ }^{35}$ Pode-se constatar que o valor encontrado neste trabalho para os compósitos MWCNT/TPU (Texin 990R) está entre os melhores descritos na literatura.
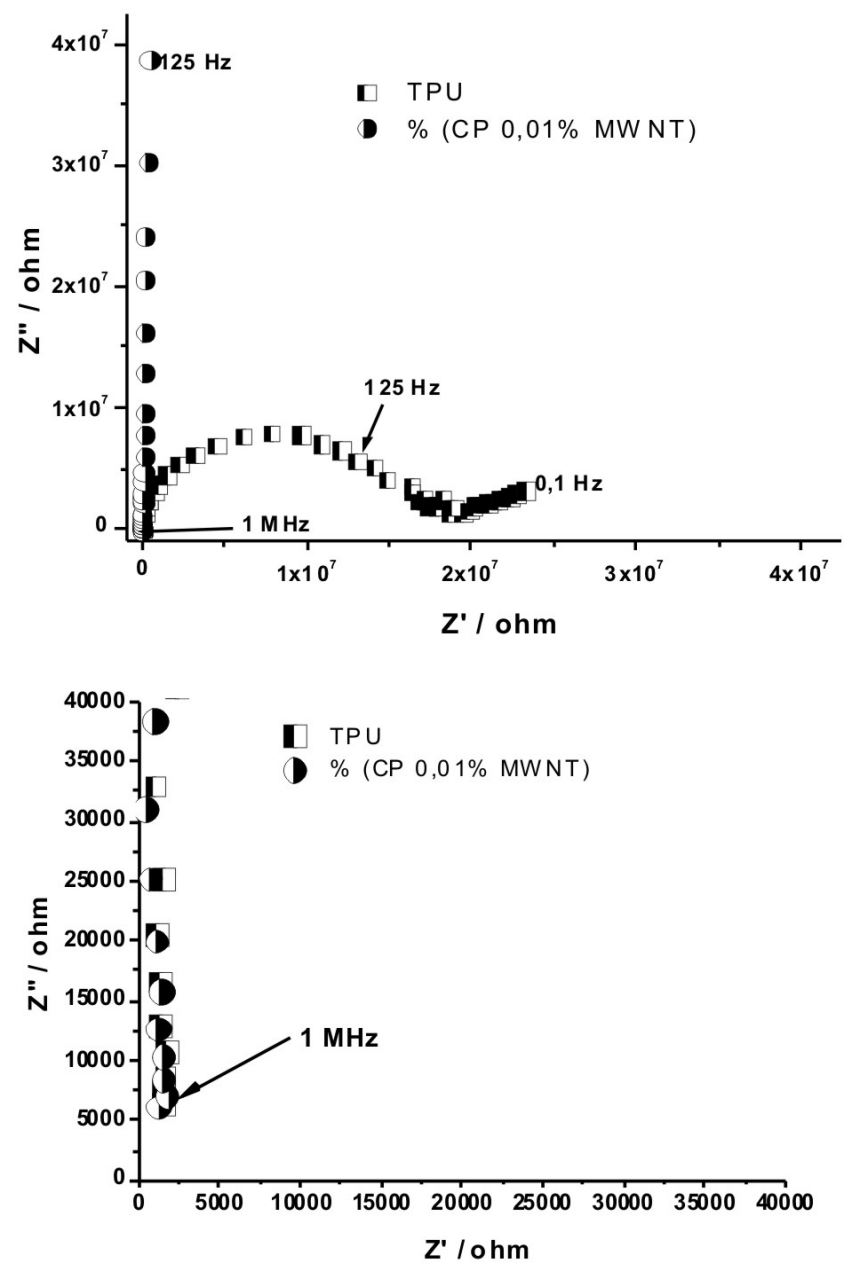

Figura 6. Diagrama de Nyquist a $35^{\circ} \mathrm{C}$ para a TPU e compósito 0,01\% $M W C N T$, mostrando a diferença entre o condutor eletrônico (CP001) e um isolante (TPU). Em destaque na figura inferior os dados em alta frequência.

A condutividade dos compósitos é bem maior que a da matriz devido à presença do MWCNT, mas ainda está abaixo do que seria esperado, considerando-se a alta condutividade dos nanotubos isolados. ${ }^{36,37}$ No entanto, esse é um fator intrínseco ao campo de compósitos. Uma carga condutora é adicionada para aumentar a condutividade da matriz isolante, que no caso dos nanocompósitos nanotubo/polímero ajuda a manter separação entre os tubos. Sem o polímero, as interações tubo-tubo seriam dominantes e, então, as partículas da carga tenderiam a se aderir e formar grandes agregados, ao invés de canais de contato entre partículas. Mas dependendo da adesão do polímero à carga, há formação de uma camada isolante em torno das partículas condutoras, diminuindo o contato entre os tubos e, em consequência, as propriedades de condução elétrica. Um balanço entre esses fatores traduz-se no aumento da condutividade da matriz,$^{38}$ mas sempre abaixo da condutividade obtida pelo contato direto entre os tubos. De fato, condutividades entre $10^{-7}-10^{-1}$ $\mathrm{S} / \mathrm{cm}$ foram descritas para nanocompósitos CNT/polímero. ${ }^{32}$ Segundo Musumeci e colaboradores, ${ }^{35}$ a maioria dos valores estão na ordem 
de $10^{-4} \mathrm{~S} / \mathrm{cm}$. Estes resultados são dependentes de vários fatores, tais como, tipo e razão de aspecto dos nanotubos, pureza da amostra, dispersão e orientação dos nanotubos na matriz e grau de recobrimento de polímero isolante sobre os nanotubos.

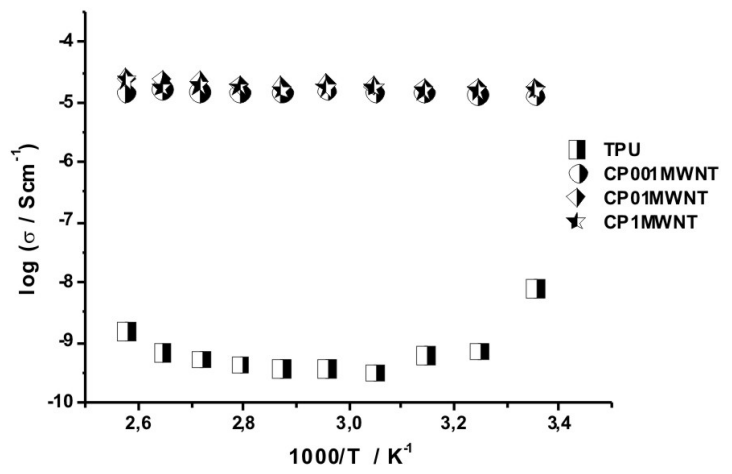

Figura 7. Diagrama de Arrhenius para a TPU e nanocompósitos MWCNT/TPU

É importante ressaltar que a amostra de MWCNT utilizada é composta por nanotubos, carbono amorfo e grafítico e partículas metálicas, como mencionado na parte experimental. Nossa experiência com este tipo de MWCNT mostra que aproximadamente 54\% em massa do material é efetivamente nanotubo. ${ }^{20}$ Carbono amorfo e grafítico organizado em nanopartículas dispersas no polímero também contribuem para a condução elétrica no material, porém apresentam razão de aspecto (comprimento sobre diâmetro) bastante inferior à dos nanotubos. Partículas esféricas (ou ovaladas) formam estruturas alongadas através do encadeamento preferencial em uma direção que permite considerar até uma razão de aspecto próxima de 10 para estes materiais. ${ }^{39}$ No caso dos nanotubos utilizados, a razão de aspecto é de 100, o que permite prever que, se apenas 54\% do material é nanotubo, a concentrações superiores a $4 \%$ em massa de nanotubos totalmente dispersos este nanocompósito atingiria o máximo de condutividade possível, como mostrado em outros trabalhos. ${ }^{35}$ De todo modo este patamar máximo de condutividade é também altamente dependente da camada isolante de polímero entre as espécies condutoras, como discutido anteriormente.

\section{CONCLUSÕES}

Duas Tg's foram determinadas para a TPU e para os compósitos através da DSC, indicando se tratar de matriz com fases separadas em domínios rígidos e flexíveis. Uma pequena variação da Tg a temperatura mais elevada, associada aos segmentos rígidos, foi observada com a introdução da amostra de MWCNTs utilizada. O estudo de EVMP em função da temperatura contribuiu para corroborar a diminuição da $\mathrm{Tg}$ produzida pela nanocarga. Ocorre um decréscimo da taxa de variação (inclinação) do aumento do volume livre com a temperatura quando os materiais ultrapassam a Tg.

As imagens de MEV mostraram boa adesão dos MWCNTs à matriz de TPU, já que não são observados espaços vazios entre os nanotubos e a poliuretana. Em paralelo, foi detectada, nos espectros de FTIR, uma perturbação das absorções de estiramento $\mathrm{C}=\mathrm{O}$ de domínios uretânicos (rígidos) quando nanotubos são adicionados à TPU.

A condutividade calculada a $25^{\circ} \mathrm{C}$ foi de cerca de $10^{-5} \mathrm{~S} / \mathrm{cm}$ para todos os nanocompósitos, indicando percolação a menos de $0,01 \%$ massa de MWCNT. Os nanotubos de carbono utilizados constituem uma amostra típica de síntese por CVD com razão de aspecto da ordem de 100 e presença de carbono amorfo, grafítico e partículas metálicas. $\mathrm{O}$ conjunto de resultados permite afirmar que foi obtido sucesso no preparo de um nanomaterial polimérico apto a dissipar carga eletrostática.

\section{MATERIAL SUPLEMENTAR}

Disponível em http://quimicanova.sbq.org.br, na forma de arquivo .PDF, com acesso livre. São apresentadas as seguintes figuras: Figura 1S. MWCNTs empregados no preparo dos compósitos; Figura 2S. Estrutura molecular da TPU; Figura 3S. Imagens dos filmes do polímero e dos compósitos CP001 a CP1; Figura 4S. a) Curva DSC típica para TPU obtida da segunda corrida de aquecimento, b) região da segunda transição vítrea na curva da TPU mostrando o método de determinação desta temperatura.

\section{AGRADECIMENTOS}

Ao CNPq, à FAPEMIG (bolsa de Pós-doutorado de R. L. Lavall), Rede Nacional de Pesquisa em Nanotubos de Carbono. À Bayer por ter gentilmente fornecido a amostra de poliuretana.

\section{REFERÊNCIAS}

1. Yu, M. F.; Lourie, O.; Dyer, M. J.; Kelly, T. F.; Ruoff, R. S.; Science 2000, 287, 637.

2. Yang, D. J.; Wang, S. G.; Zhang, Q.; Sellin, P. J.; Chen, G.; Phys. Lett. A 2004, 329, 207.

3. Moniruzzaman, M.; Winey, K. I.; Macromolecules 2006, 39, 5194.

4. Ajayan, P. M.; Tour, J. M.; Nature 2007, 447, 1066.

5. Ramasubramaniam, R.; Chen, J.; Liu, H. ; Appl. Phys. Lett. 2003, 83, 2928.

6. http://www.fibrils.com, acessada em Novembro 2009.

7. Liu, P.; Eur. Polym. J. 2005, 41, 2693.

8. Smalley, R. E.; Colbert, D. T.; Smith, K. A.; O'Connell, M.; US Pat. 7008563 B2 2006

9. Chakrabarti, K.; Nambissan, P. M. G.; Mukherjee, C. D.; Bardhan, K. K.; Kim, C.; Yang, K. S.; Carbon 2006, 44, 948.

10. Jean, Y. C.; Mellon, P. E.; Schrader, D. M., eds.; Principles and Applications of Positron \& Positronium Chemistry, World Scientific Publishing Company: Singapore, 2003

11. Machado, J. C.; Silva, G. G.; Oliveira, F. C.; Lavall, R. L.; Rieumont, J.; Licinio, P.; Windmoller, D.; J. Polym. Sci., Part B: Polym. Phys. 2007, 45, 2400.

12. Patricio, P. S.; Silva, G. G.; Machado, J. C.; J. Appl. Polym. Sci. 2007, 105, 641.

13. Chakrabarti, K.; Nambissan, P. M. G.; Mukherjee, C. D.; Bardhan, K. K.; Kim, C.; Yang, K. S.; Carbon 2007, 45, 2777.

14. de Sales, J. A.; Patricio, P.; Windmoller, D.; Silva, G. G.; Machado, J. C.; J. Membr. Sci. 2008, 310, 129.

15. http://www.bayer.com.br, acessada em Novembro 2009.

16. Andrews, R.; Jacques, D.; Qian, D.; Rantell, T. ; Acc. Chem. Res. 2002, 35,1008 .

17. Schnitzler, M. C.; Oliveira, M. M.; Ugarte, D.; Zarbin, A. J. G .; Chem. Phys. Lett., 2003, 381, 541.

18. Dillon, A. C.; Gennett, T.; Jones, K. M.; Alleman, J. L.; Parilla, P. A.; Heben, M. J.; Adv. Mater. 1999, 11, 1354.

19. Souza Filho, A. G.; Fagan, S. B.; Quim. Nova 2007, 30, 1695.

20. Trigueiro, J. P. C.; Silva, G. G.; Lavall, R. L.; Furtado, C. A.; Oliveira, S.; Ferlauto, A. S.; Lacerda, R. G.; Ladeira, L. O.; Liu, J-W.; Frost, R. L.; George, G. A.; J. Nanosci. Nanotechnol. 2007, 7, 3477.

21. Kirkegaard, P.; Eldrup, M.; Comput. Phys. Commun. 1974, 7, 401.

22. Tao, S. J.; J. Chem. Phys. 1972, 56, 5499.

23. Nakanishi, H.; Wang, S. J.; Jean, Y. C. Em Positron Annihilation Studies of Fluids; Sharma, S. C., ed.; World Scientific: Singapore, 1988, p.292.

24. Shvartzaman-Cohen, R.; Levi-Kalisman, Y.; Nativ-Roth, E.; YerushalmiRozen, R.; Langmuir 2004, 20, 6085.

25. Koerner, H.; Kelley, J. J.; Vaia, R. A.; Macromolecules 2008, 41, 4709. 
26. van Heumen, J. D.; Stevens, J. R.; Macromolecules 1995, 28, 4268.

27. Digar, M.; Wen, T-C.; Polymer 2001, 42, 71.

28. Wen, T-C.; Du, Y-L.; Digar, M.; Eur. Polym. J. 2002, 38, 1039.

29. Wen, T-C.; Kuo, H-H.; Gopalan, A.; Solid State Ionics 2002, 147, 171.

30. Lee, S-M.; Chen, C-Y.; Wang, C-C.; Huang, Y-H.; Electrochim. Acta 2003, 48, 669 .

31. Wang, Y. Y.; Nakanishi, H.; Jean, Y. C.; Sandreczki, T. C.; J. Polym. Sci., Part B: Polym. Phys. 1990, 28, 1431.

32. Dalmas, F.; Dendievel, R.; Chazeau, L.; Cavaillé, J-Y.; Gauthier, C.; Acta Mater. 2006, 54, 2923.

33. Sandler, J. K. W.; Kirk, J. E.; Kinloch, I. A.; Shaffer, M. S. P.; Windle, A. H.; Polymer 2003, 44, 5893.

34. Koerner, H.; Liu, W.; Alexander, M.; Mirau, P.; Dowty, H.; Vaia, R. A.; Polymer 2005, 46, 4405.
35. Musumeci, A. W.; Silva, G. G.; Liu, J-W.; Martens, W. N.; Waclawik, E. R.; Polymer 2007, 48, 1667.

36. Ajayan, P. M.; Chem. Rev. 1999, 99, 1787.

37. Barrau, S.; Demont, P.; Peigney, A.; Laurent, C. ; Lacabanne, C. ; Macromolecules 2003, 36, 5187.

38. Gubbels, F.; Blacher, S.; Vanlathem, E.; Jérôme, R.; Deltour, R.; Brouers, F.; Teyssié, Ph. ; Macromolecules 1995, 28, 1559.

39. Lavall, R. L.; Borges, R. S.; Calado, H. D. R.; Welter, C.; Trigueiro, J. P. C.; Rieumont, J.; Neves, B. R. A.; Silva, G. G.; J. Power Sources 2008, 177,652 .

40. Silverstein, R. M.; Webster, F. X.; Identificação Espectrométrica de Compostos Orgânicos, $6^{\mathrm{a}}$ ed., LTC: Rio de Janeiro, 2000. 


\section{NANOCOMPÓSITOS DE POLIURETANA TERMOPLÁSTICA E NANOTUBOS DE CARBONO DE PAREDES MÚLTIPLAS PARA DISSIPAÇÃO ELETROSTÁTICA}

Rodrigo L. Lavall, Juliana A. de Sales, Raquel S. Borges, Hallen D. R. Calado, José C. Machado, Dario Windmöller e Glaura G. Silva*

Departamento de Química, Instituto de Ciências Exatas, Universidade Federal de Minas Gerais. Av. Antônio Carlos, 6627, $31270-$ 901 Belo Horizonte - MG, Brasil

Rodrigo G. Lacerda e Luiz O. Ladeira

Departamento de Física, Instituto de Ciências Exatas, Universidade Federal de Minas Gerais. Av. Antônio Carlos, 6627, 31270 901 Belo Horizonte - MG, Brasil

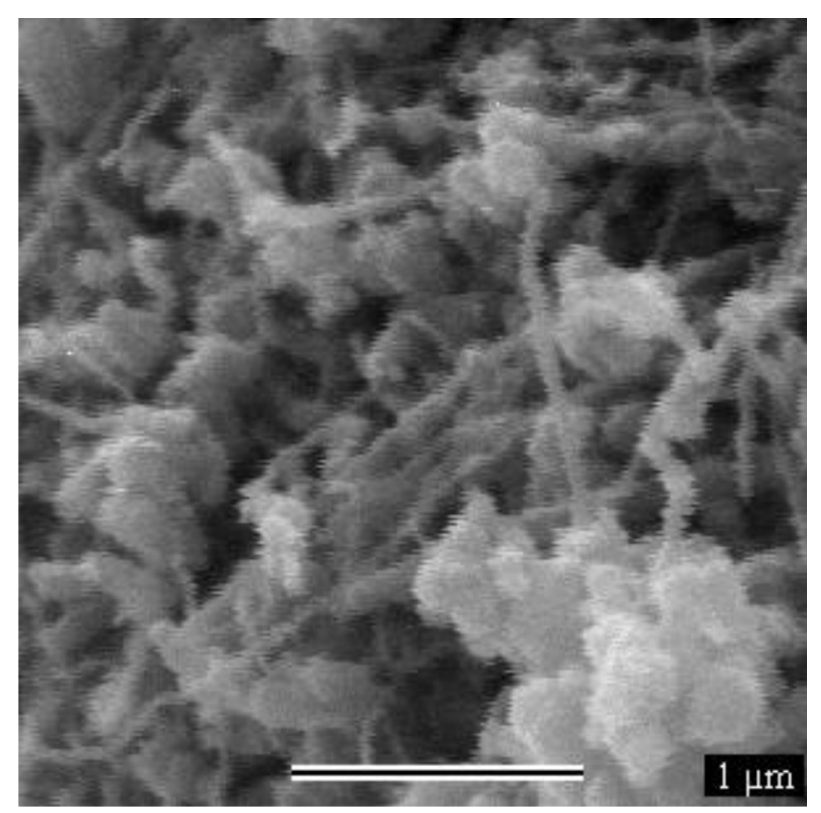

Figura 1S. MWCNTs empregados no preparo dos compósitos

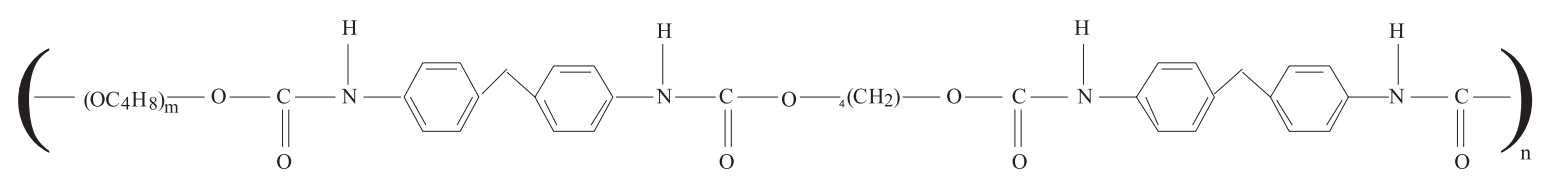

Figura 2S. Estrutura molecular da TPU

*e-mail: glaura@qui.ufmg.br 
UFMG/Grupo de Materiais M ulticomponentes de Matriz Pol imérica
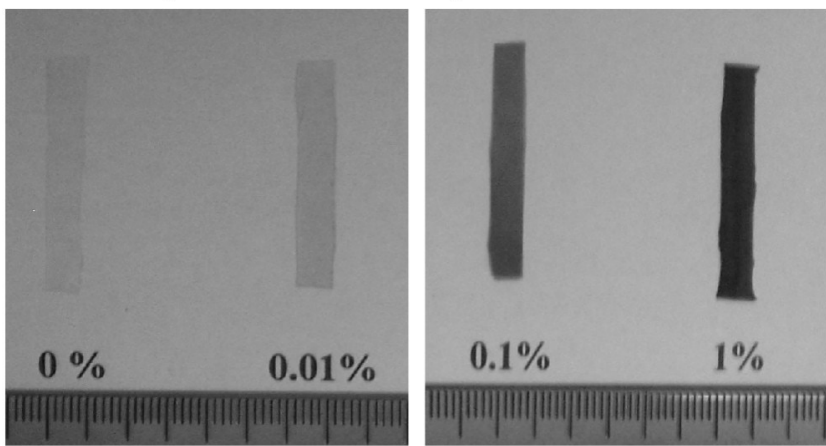

Figura 3S. Imagens dos filmes do polímero e dos compósitos CP001 a CP1

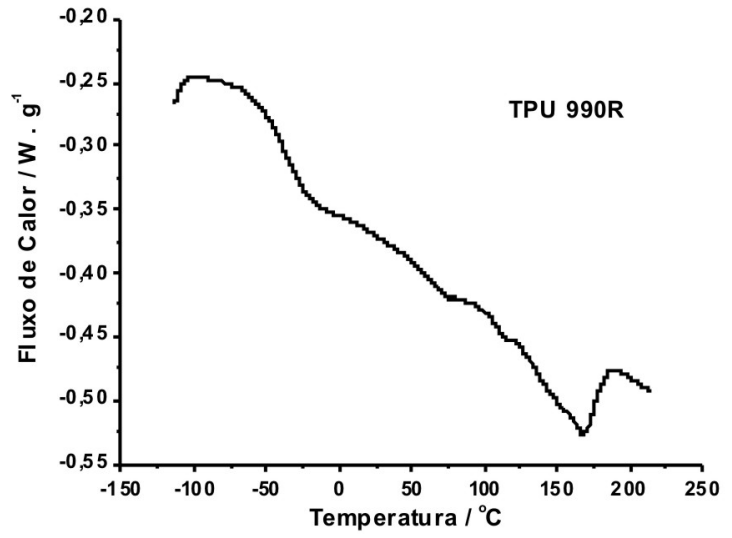

(a)

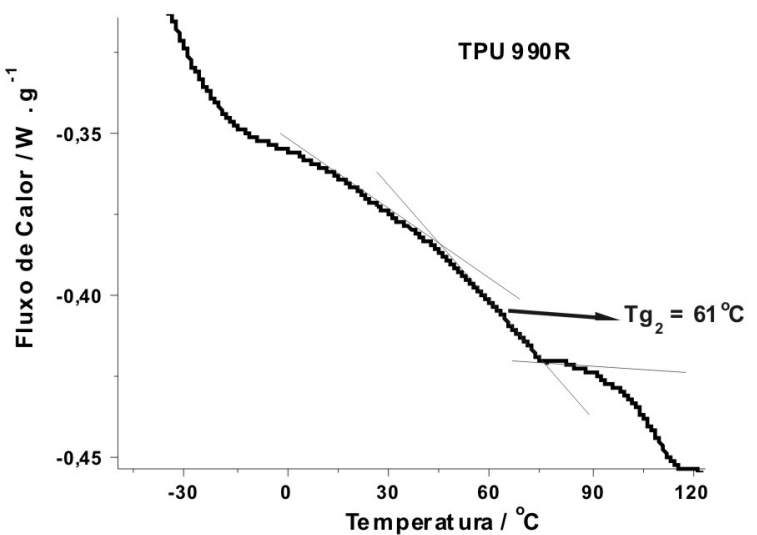

(b)

Figura 4S. a) Curva DSC típica para TPU obtida da segunda corrida de aquecimento, b) região da segunda transição vítrea na curva da TPU mostrando o método de determinação desta temperatura 